\title{
High energy leptonic collisions and electroweak parton distribution functions
}

\author{
Tao Han $\odot,{ }^{*}$ Yang Ma $\odot,^{\dagger}$ and Keping Xie $\odot^{*}$ \\ PITT PACC, Department of Physics and Astronomy, University of Pittsburgh, \\ 3941 O'Hara Street, Pittsburgh, Pennsylvania 15260, USA
}

(Received 28 August 2020; revised 16 November 2020; accepted 26 January 2021; published 18 February 2021)

\begin{abstract}
In high-energy leptonic collisions well above the electroweak scale, the collinear splitting mechanism of the electroweak gauge bosons becomes the dominant phenomena via the initial state radiation and the final state showering. We point out that at future high-energy lepton colliders, such as a multi-TeV muon collider, the electroweak parton distribution functions (EW PDFs) should be adopted as the proper description for partonic collisions of the initial states. The leptons and electroweak gauge bosons are the EW partons, that evolve according to the unbroken Standard Model (SM) gauge group and that effectively resum potentially large collinear logarithms. We present a formalism for the EW PDFs at the leading-log (LL) accuracy. We calculate semi-inclusive cross sections for some important SM processes at a future multi-TeV muon collider. We conclude that it is appropriate to adopt the EW PDF formalism for future high-energy lepton colliders.
\end{abstract}

DOI: 10.1103/PhysRevD.103.L031301

\section{INTRODUCTION}

With the discovery of the Higgs boson at the CERN Large Hadron Collider (LHC), the particle spectrum of the Standard Model (SM) is complete. The next target at the energy frontier will be to study the Higgs properties and to search for the next scale beyond the SM [1]. The physics potential for TeV-scale $e^{+} e^{-}$linear colliders, such as the International Linear Collider [2] and the CERN Compact Linear Collider (CLIC) [3], has been studied to great details. More recently, due to the breakthrough in the cooling technology for a muon beam [4], there has been renewed interest in constructing a $\mu^{+} \mu^{-}$collider reaching a centerof-momentum energy (c.m. energy) $\sqrt{s} \sim \mathcal{O}(10 \mathrm{TeV})$. Advancement of the wake-field electron acceleration technology [5] has also been encouraging to have stimulated our ambition for reaching multi-TeV threshold in leptonic collisions.

Lepton colliders provide a clean experimental environment for precision measurements of physical observables and for discovery of new particles. Near a mass threshold, the $e^{+} e^{-}$annihilation may produce a new particle singly in

\footnotetext{
than@pitt.edu

mayangluon@pitt.edu

*xiekeping@pitt.edu
}

Published by the American Physical Society under the terms of the Creative Commons Attribution 4.0 International license. Further distribution of this work must maintain attribution to the author(s) and the published article's title, journal citation, and DOI. Funded by SCOAP. the $s$-channel, or a particle/anti-particle in pair. As the beam energy increases, the initial state radiation (ISR) becomes substantial. It not only degrades the colliding energies of the leptons, but also generates new reactions of the radiation fields. The most familiar phenomenon is the collinear photon radiation off the high energy charged particles given by the Weizsäicker-Williams spectrum $[6,7]$

$$
\mathcal{P}_{\gamma, \ell}(x) \approx \frac{\alpha}{2 \pi} P_{\gamma, \ell}(x) \ln \frac{E^{2}}{m_{\ell}^{2}},
$$

where the splitting functions are $P_{\gamma / \ell}(x)=\left(1+(1-x)^{2}\right) / x$ for $\ell \rightarrow \gamma$ and $P_{\ell / \ell}(x)=\left(1+x^{2}\right) /(1-x)$ for $\ell \rightarrow \ell$, with an energy $x E$ off the charged lepton beam of energy $E$. This is the leading order effective photon approximation (EPA).

In Fig. 1, we show some representative production cross sections versus the $\mu^{+} \mu^{-}$c.m. energy $\sqrt{s}$ for

$$
\mu^{+} \mu^{-} \rightarrow W^{+} W^{-}, Z Z, t \bar{t}, Z H \quad \text { and } \quad t \bar{t} H .
$$

The dashed (falling) curves are for the direct $\mu^{+} \mu^{-}$ annihilation, and the solid curves (slightly above the dashed) include the ISR effects [8]. We see the typical fall of the annihilation cross sections as $1 / s$. The ISR reduces the c.m. energy at the collision and thus increases the cross section. At $\sqrt{s}=10 \mathrm{TeV}(30 \mathrm{TeV})$, the cross section for $\mu^{+} \mu^{-} \rightarrow t \bar{t}$ production can be enhanced by $40 \%$ $(60 \%)$ due to the ISR effects. Owing to the collinear enhancement, the two-photon $(\gamma \gamma)$ fusion processes grow 


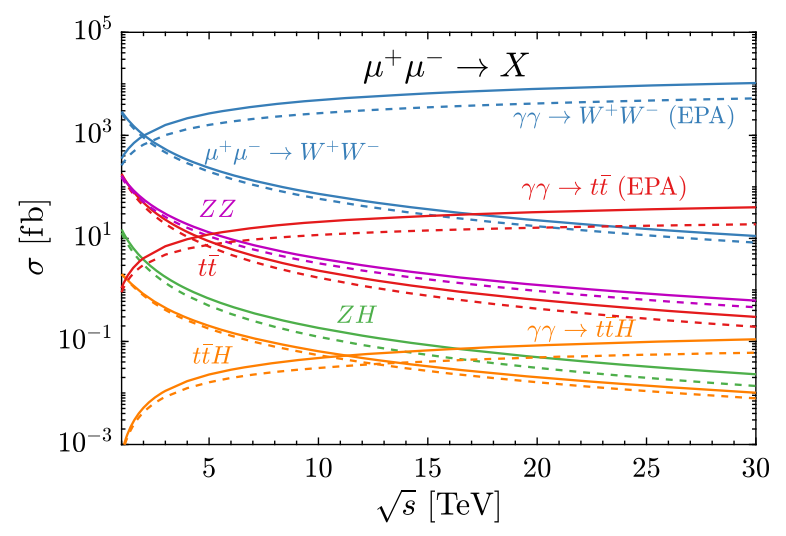

FIG. 1. Production cross sections in $\mu^{+} \mu^{-}$collisions versus the c.m. energy. The dashed falling curves are for the direct $\mu^{+} \mu^{-}$ annihilation, and the solid falling curves (slightly above the dashed) include the ISR effects. The solid rising curves are for $\gamma \gamma$ EPA by Eq. (1) and the dashed rising curves are from the leadingorder $\gamma$-PDF at $Q=\sqrt{\hat{s}} / 2$.

double-logarithmically. We calculate the total cross sections with the EPA spectrum in Eq. (1) for

$$
\gamma \gamma \rightarrow W^{+} W^{-}, \bar{t} \bar{t} \text { and } \bar{t} \bar{t} H
$$

Those processes take over the annihilation channels at higher energies $\sqrt{s} \approx 2.5,4.5,11 \mathrm{TeV}$ for $W^{+} W^{-}, t \bar{t}$ and $\bar{t} \bar{t} H$ production, respectively, as shown in Fig. 1 by the rising solid curves labeled by EPA. At $\sqrt{s} \approx 30 \mathrm{TeV}$, the production rate for $\gamma \gamma \rightarrow t \bar{t}$ is higher by two orders of magnitude than that for $\mu^{+} \mu^{-} \rightarrow t \bar{t}$ annihilation.

However, this description becomes inadequate at some high scales. First, at high energies $E \gg m_{\ell}$, the collinear $\operatorname{logarithm}(\alpha / 2 \pi) \ln \left(E^{2} / m_{\ell}^{2}\right)$ may be sizeable and needs to be resummed for reliable predictions. This leads to the QED analogue of the Dokshitzer-Gribov-Lipatov-AltarelliParisi (DGLAP) equations [9-11], the concept of QED parton distribution functions (PDFs) for the photon and charged fermions [12-14]. To estimate the resummation effects, we plot the cross sections with the leading-order $\gamma$ PDF with a scale $Q=\sqrt{\hat{s}} / 2$, where $\sqrt{\hat{s}}$ is the $\gamma \gamma$ c.m. energy. As shown in Fig. 1 by the dashed rising curves below those of EPA, we see that the rates are lowered as expected, and could be smaller by about a factor of two at $30 \mathrm{TeV}$.

More importantly, as pointed out in Refs. [15-17] and explored in details [18], at scales $Q^{2} \gg M_{Z}^{2}$, the SM gauge symmetry $\mathrm{SU}(2)_{L} \otimes \mathrm{U}(1)_{Y} \quad$ is effectively restored. Consequently, the four EW gauge bosons $\left(W^{ \pm, 3}, B\right)$ in the SM must be taken into account all together coherently with $B-W^{3}$ mixing and interference. The fermion interactions are chiral and the couplings and states evolve according to the SM unbroken gauge symmetry. One needs to invoke the picture of electroweak parton distribution functions (EW PDFs) [19-21] dynamically generated by the electroweak and Yukawa interactions. The longitudinally polarized gauge bosons capture the remnants of the EW symmetry breaking. The effects are governed by power corrections of the order $M_{Z}^{2} / Q^{2}$ [22,23], a measure of the Goldstone-boson equivalence violation [15,24], analogous to higher-twist effects in QCD.

\section{ELECTROWEAK PARTON DISTRIBUTION FUNCTIONS}

Below the EW scale $Q<M_{Z}$, the effects of the $\mathrm{SU}(2)_{L}$ gauge bosons are suppressed by $g^{2} / M_{Z}^{2}$. The gauge boson radiation off a charged lepton beam $\left(\ell^{ \pm}=e^{ \pm}, \mu^{ \pm}\right)$is essentially purely electromagnetic. At the EW scale and above, all electroweak states in the unbroken SM are dynamically activated. The massless states involved at the leading order are

$$
\ell_{R}, \ell_{L}, \nu_{L} \quad \text { and } B, W^{ \pm, 3} .
$$

We will not include the Higgs sector in the initial state partons since the Yukawa couplings to $e, \mu$ are not relevant for the current consideration. However, we must include the effects of longitudinally polarized gauge bosons characterized by power corrections of the order $M_{Z}^{2} / Q^{2}$. Denote an EW PDF as $f_{i}\left(x, Q^{2}\right)$ with $i$ labeling a particle with an energy fraction $x$ at a factorization scale $Q$. The EW PDFs evolve according to the full EW DGLAP equations [16,25]

$$
\frac{\mathrm{d} f_{i}}{\mathrm{~d} \ln Q^{2}}=\sum_{I} \frac{\alpha_{I}}{2 \pi} \sum_{j} P_{i, j}^{I} \otimes f_{j}
$$

where $I$ specifies the gauge group, and the $P_{i j}^{I}$ are the splitting functions for $j \rightarrow i$. The complete list of the EW splitting functions for the SM chiral states are available in Refs. $[15,16,20]$. The initial condition for a lepton beam is $f_{\ell}\left(x, m_{\ell}^{2}\right) \approx \delta(1-x)+\mathcal{O}(\alpha)$ and it evolves as $\ln \left(Q^{2} / m_{\ell}^{2}\right)$. At the electroweak scale, the matching conditions are $f_{\gamma}\left(x, M_{Z}^{2}\right) \neq 0, f_{Z}\left(x, M_{Z}^{2}\right)=0, f_{\gamma Z}\left(x, M_{Z}^{2}\right)=0$, with a general relation

$$
\left(\begin{array}{c}
f_{B} \\
f_{W^{3}} \\
f_{B W^{3}}
\end{array}\right)=\left(\begin{array}{ccc}
c_{W}^{2} & s_{W}^{2} & -c_{W} s_{W} \\
s_{W}^{2} & c_{W}^{2} & c_{W} s_{W} \\
2 c_{W} s_{W} & -2 c_{W} s_{W} & c_{W}^{2}-s_{W}^{2}
\end{array}\right)\left(\begin{array}{c}
f_{\gamma} \\
f_{Z} \\
f_{\gamma Z}
\end{array}\right),
$$

where $s_{W}=\sin \theta_{W}$ is the weak mixing angle. The mixed $\operatorname{PDF} f_{\gamma Z}$ (or $f_{B W^{3}}$ ) represents a mix state and is important to account for the interference between the diagrams involving $\gamma / Z$ (or $B / W^{3}$ ) $[15,16,19]$. Chiral couplings and their RGE running are fully taken into account including the correlation between the polarized PDFs and the corresponding polarized scattering amplitudes. With one-loop virtual corrections, our results are accurate at the leading$\log$ (LL) order. In Fig. 2(a), we present EW PDFs for the 

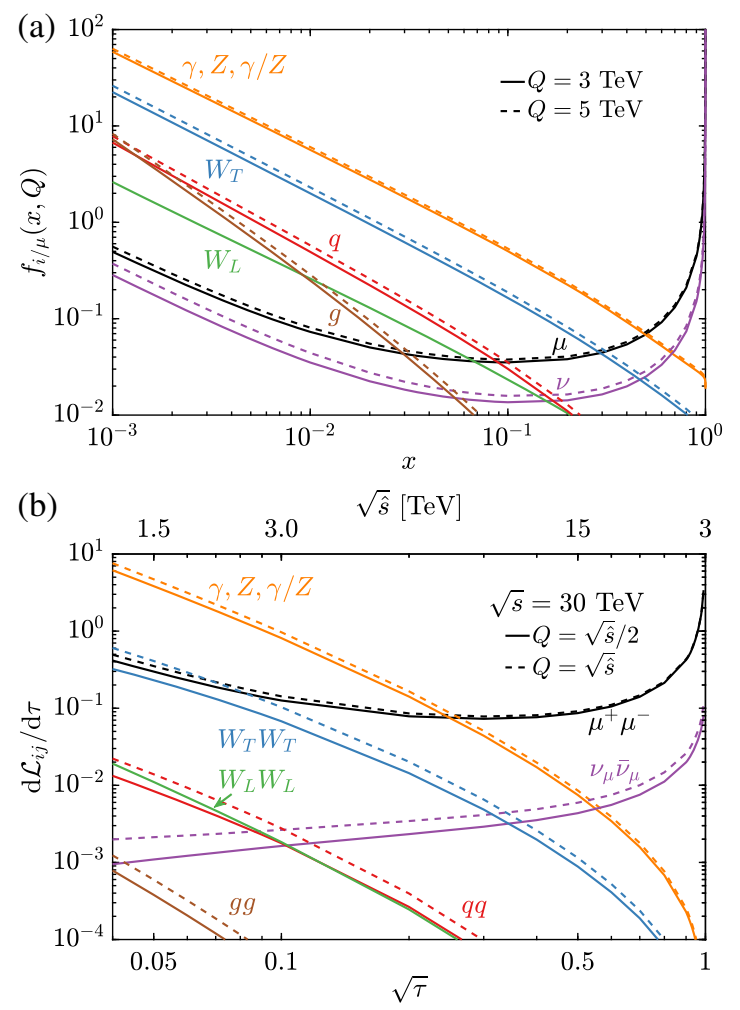

FIG. 2. Distributions for (a) EW PDFs $f_{i}(x)$ and, (b) parton luminosities $\mathrm{d} \mathcal{L}_{i j} / \mathrm{d} \tau$ versus $\sqrt{\tau}$ for $\sqrt{s}=30 \mathrm{TeV}$ with a factorization scale $Q=\sqrt{\hat{s}} / 2$ (solid) and $\sqrt{\hat{s}}$ (dashed).

states in Eq. (4) for $\ell=\mu$ with a scale $Q=3 \mathrm{TeV}$ and $5 \mathrm{TeV}$. For completeness, we have also included the quarks $q=\sum_{i=d}^{t}\left(q_{i}+\bar{q}_{i}\right)$ and gluons from the higher-order splittings. We give the averaged momentum fractions $\left\langle x f_{i}\right\rangle=\int x f_{i}(x) \mathrm{d} x$ carried by various parton species in Table I. The two scale choices lead to less than $20 \%$ difference for the EW PDFs. As expected, the fermionic states sharply peak at $x \approx 1$, while the bosonic states peak at $x \approx 0$, reflecting the infrared behavior. It is noted that there is an enhanced rate at small $x$ for the fermions, deviating from the leading order behavior $\sim 1 /(1-x)$. This is from the soft $\gamma^{*} / Z^{*} / W^{*}$ splitting at higher orders. Owing to the large flux of photons at low scales, the neutral EW PDFs are largest. Unlike all the other EW PDFs that scale logarithmically with $Q$, the longitudinal gauge bosons $\left(W_{L}, Z_{L}\right)$ do not scale with $Q$ at the leading order

TABLE I. Momentum fractions (\%) carried by various parton species. The sea leptons include $\ell_{\text {sea }}=\bar{\mu}+\sum_{i \neq \mu}\left(\ell_{i}+\bar{\ell}_{i}\right)$ and $\nu=\sum_{i}\left(\nu_{i}+\bar{\nu}_{i}\right)$. The quark components include all the 6 flavors.

\begin{tabular}{lccccccc}
\hline \hline$Q$ & $\mu$ & $\gamma, Z, \gamma Z$ & $W^{ \pm}$ & $\nu$ & $\ell_{\text {sea }}$ & $q$ & $g$ \\
\hline$M_{Z}$ & 97.9 & 2.06 & 0 & 0 & 0.028 & 0.035 & 0.0062 \\
$3 \mathrm{TeV}$ & 91.5 & 3.61 & 1.10 & 3.59 & 0.069 & 0.13 & 0.019 \\
$5 \mathrm{TeV}$ & 89.9 & 3.82 & 1.24 & 4.82 & 0.077 & 0.16 & 0.022 \\
\hline \hline
\end{tabular}

$[15,16,26]$-an explicit example for Bjorken-scaling restoration.

\section{CROSS SECTIONS FOR SEMI-INCLUSIVE PROCESSES IN $\mu^{+} \mu^{-}$COLLISIONS}

We write the production cross section of an exclusive final state $F$ and the unspecified remnants $X$ in terms of the parton luminosity $\mathrm{d} \mathcal{L}_{i j} / \mathrm{d} \tau$ and the corresponding partonic subprocess cross section $\hat{\sigma}$

$$
\begin{aligned}
& \sigma\left(\ell^{+} \ell^{-} \rightarrow F+X\right)=\int_{\tau_{0}}^{1} \mathrm{~d} \tau \sum_{i j} \frac{\mathrm{d} \mathcal{L}_{i j}}{\mathrm{~d} \tau} \hat{\sigma}(i j \rightarrow F), \\
& \frac{\mathrm{d} \mathcal{L}_{i j}}{\mathrm{~d} \tau}=\frac{1}{1+\delta_{i j}} \int_{\tau}^{1} \frac{\mathrm{d} \xi}{\xi}\left[f_{i}\left(\xi, Q^{2}\right) f_{j}\left(\frac{\tau}{\xi}, Q^{2}\right)+(i \leftrightarrow j)\right],
\end{aligned}
$$

where $\tau=\hat{s} / s$ with $\sqrt{s}(\sqrt{\hat{s}})$ the collider (parton) c.m. energy. The production threshold is $\tau_{0}=m_{F}^{2} / s$.

In presenting our results for production of SM particles at a high-energy lepton collider, for definitiveness, we consider a future $\mu^{+} \mu^{-}$collider with multi-TeV energies. It is informative to first examine the parton luminosities as shown in Fig. 2(b) for $\sqrt{s}=30 \mathrm{TeV}$ versus $\sqrt{\tau}$, with a variety of partonic initial states. The upper horizontal axis labels the accessible $\sqrt{\hat{s}}$. Although we properly evolve the EW PDFs according to the unbroken SM gauge groups, we convert the states back for the sake of common intuition, shown in the figure for $\mu^{+} \mu^{-}, \nu_{\mu} \bar{\nu}_{\mu}, \gamma \gamma / Z Z / \gamma Z, W_{T} W_{T}$ and $W_{L} W_{L}$. We see that the fermionic luminosities peak near the machine c.m. energy $\tau \approx 1$, while the gauge boson luminosities, generically called vector boson fusion (VBF) dominate at lower partonic energy $\sqrt{\hat{s}}$. As noted earlier, the neutral gauge boson luminosities are the largest, followed by $W_{T}$ and $W_{L}$.

We emphasize the "inclusiveness" of the production processes. For example, for an exclusive final state of $t \bar{t}$ production, one needs to sum over all the observationally indistinguishable partonic contributions in the initial state $\mu^{+} \mu^{-}, \gamma \gamma, \gamma Z, Z Z, W^{+} W^{-} \rightarrow t \bar{t}$. Contributions from the quark and gluon initial states are sub-leading as seen in the parton luminosities in Fig. 2(b), and we do not include them in the cross section calculations throughout this paper. Since the collinear remnants are not observationally resolved, one cannot separate the $\mu^{+} \mu^{-} / \nu_{\mu} \bar{\nu}_{\mu}$ annihilations from the VBF. For this reason, we call such processes, i.e., $\mu^{+} \mu^{-} \rightarrow t \bar{t}$ "semi-inclusive." This is analogous to the $t \bar{t}$ production at hadron colliders from the partonic subprocesses $q \bar{q}, g g \rightarrow t \bar{t}$.

In Fig. 3(a), we show the semi-inclusive production cross sections at a $\mu^{+} \mu^{-}$collider versus the collider c.m. energy $\sqrt{s}$ from $1 \mathrm{TeV}$ to $30 \mathrm{TeV}$. We choose the factorization 

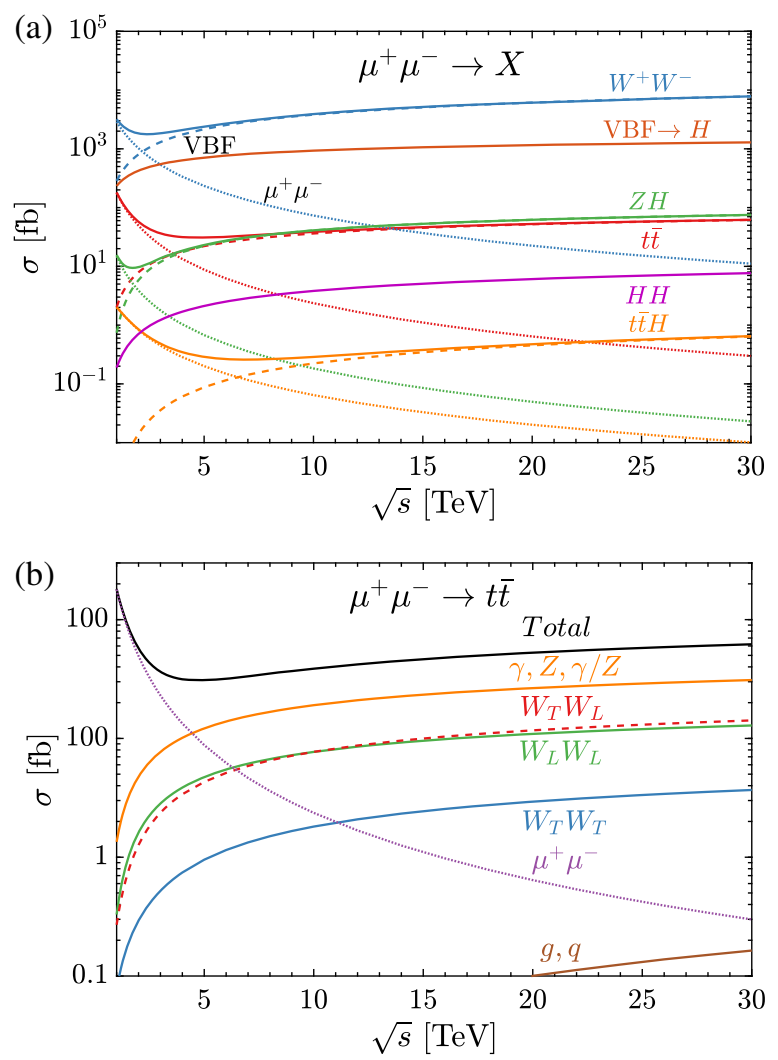

FIG. 3. Production cross section for semi-inclusive processes at a $\mu^{+} \mu^{-}$collider versus the c.m. energy. The solid curves are for (a) the total cross sections and the dashed (dotted) curves from $\operatorname{VBF}\left(\mu^{+} \mu^{-}\right.$annihilation) with $\mathrm{EW}$ PDF, and (b) for $t \bar{t}$ production decomposed to the underlying contributions from $\mu^{+} \mu^{-}, \gamma / Z / \gamma Z, W_{T} W_{L}, W_{L} W_{L}$ and $W_{T} W_{T}$.

scale $Q=\sqrt{\hat{s}} / 2$ in calculating the EW PDFs. ${ }^{1}$ The solid curves are the total cross sections for the semi-inclusive processes for

$$
\mu^{+} \mu^{-} \rightarrow W^{+} W^{-}, H, Z H, t \bar{t}, H H \quad \text { and } \quad t \bar{t} H,
$$

combining the contributions from both fermionic initial states and the VBF. We indicate the VBF contributions by the dashed curves, ${ }^{2}$ and the fermionic contributions by the dotted curves, respectively, below the solid curves. It is important to note that, although there is no logarithmic evolution for the $W_{L}$ PDF, the partonic subprocess cross sections are much enhanced for $W_{L} W_{L}, Z_{L} Z_{L} \rightarrow t \bar{t}, t \bar{t} H$ and $H, Z H, H H$, due to the Goldstone-boson interactions.

\footnotetext{
${ }^{1}$ To validate the EW PDF approximation, we have imposed an angular cutoff for the $W / Z$ initiated processes in the c.m. frame $\cos \theta<1-m^{2} / \hat{s}$, where $m$ is the relevant particle mass involved in the process. We have included a tighter cut $\cos \theta<0.99$ and $\sqrt{\hat{s}}>500 \mathrm{GeV}$ for the $W^{+} W^{-}, Z H$ final states.

${ }^{2}$ Many of the VBF processes have been calculated recently in Ref. [27] at the tree-level. We have good agreements with theirs where ever they overlap.
}
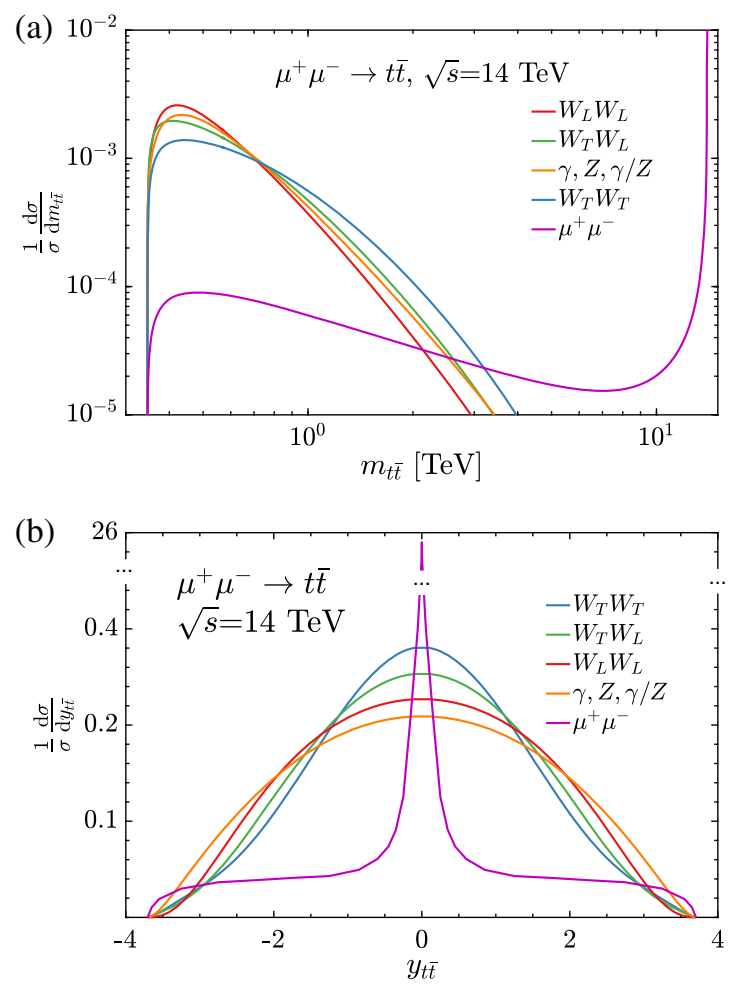

FIG. 4. Normalized differential distributions for the final state $t \bar{t}$ system (a) the invariant mass $m_{t \bar{t}}$ and (b) the rapidity $y_{t \bar{t}}$.

The VBF processes take over the annihilation channels at higher energies $\sqrt{s} \approx 2.3,3.5,6.5 \mathrm{TeV}$ for $W^{+} W^{-}, t \bar{t}$ and $t \bar{t} H$, respectively. To appreciate the individual contributions from the underlying partonic subprocesses, we decompose them for the process $\mu^{+} \mu^{-} \rightarrow t \bar{t}$ versus the c.m. energy, as shown in Fig. 3(b) for $\mu^{+} \mu^{-}, \gamma \gamma / \gamma Z / Z Z, W_{T} W_{L}, W_{L} W_{L}$ as well as $W_{T} W_{T}$. As expected, the QED contribution remains to be the leading channel. Not well appreciated, the $W_{T} W_{L} / W_{L} W_{L}$ contributions become as significant.

We now examine the kinematic distributions for the final state $t \bar{t}$ system, for the individual contributions $\mu^{+} \mu^{-}, \gamma / Z, W_{T} W_{L}, W_{L} W_{L}$ and $W_{T} W_{T}$. Shown in Fig. 4(a) are the normalized invariant mass distributions $m_{t \bar{t}}$. We see that, for the $\mu^{+} \mu^{-}$annihilation, the distribution is sharply peaked at the collider c.m. energy, with a tail due to the radiative return. For the VBF, they are peaked after the $2 m_{t}$ threshold. We show in Fig. 4(b) the normalized rapidity distributions of the system $y_{t \bar{t}}$. Again, events from the $\mu^{+} \mu^{-}$annihilation are sharply central, while those from VBF are spread out, reflecting the boost due to the momentum imbalance between the two incoming partons.

We summarize our results utilizing the EW PDFs in Table II for a few characteristic processes for a muon collider with a few representative energies 3, 6, 10, 14 and $30 \mathrm{TeV}$. For the sake of illustration, we once again separate the partonic sub-processes by the fermionic annihilation and by VBF. 
TABLE II. Production cross sections at a muon collider in units of fb by VBF utilizing the EW PDF and by direct $\mu^{+} \mu^{-}$annihilation with ISR effects.

\begin{tabular}{lcccccccccc}
\hline \hline$\sqrt{s}(\mathrm{TeV})$ & \multicolumn{3}{c}{3} & \multicolumn{2}{c}{6} & \multicolumn{2}{c}{10} & \multicolumn{2}{c}{14} & \multicolumn{2}{c}{30} \\
$\sigma(\mathrm{fb})$ & $\mathrm{VBF}$ & $\mu \mu$ & $\mathrm{VBF}$ & $\mu \mu$ & $\mathrm{VBF}$ & $\mu \mu$ & $\mathrm{VBF}$ & $\mu \mu$ & $\mathrm{VBF}$ & $\mu \mu$ \\
\hline$W^{+} W^{-}$ & 1300 & 540 & 2500 & 170 & 3800 & 73 & 4900 & 41 & 7800 & 11 \\
$t \bar{t}$ & 13 & 23 & 25 & 6.2 & 36 & 2.4 & 43 & 1.3 & 61 & 0.30 \\
$Z H$ & 12 & 1.8 & 26 & 0.48 & 41 & 0.18 & 51 & 0.097 & 75 & 0.023 \\
$H H$ & 1.2 & $\ldots$ & 2.5 & $\ldots$ & 3.8 & $\ldots$ & 4.8 & $\ldots$ & 7.6 & $\ldots$ \\
$t \bar{t} H$ & 0.036 & 0.45 & 0.12 & 0.15 & 0.22 & 0.065 & 0.32 & 0.037 & 0.64 & 0.010 \\
\hline \hline
\end{tabular}

\section{DISCUSSIONS AND CONCLUSION}

(a) The naive EPA is inadequate at high scales. The QED evolution of $\ln \left(Q^{2} / m_{\ell}^{2}\right)$ in the $\gamma$-PDF should capture the dominant effect at an appropriate physical scale $Q^{2}$. Although the $Z$ contribution is typically small until reaching a very high scale, the mixed state $\gamma Z\left(B W^{3}\right)$ needs to be taken into account that often interferes destructively.

(b) The EW PDF approach allows for calculating individual contributions from the polarized initial state partons, with correlations to the corresponding subprocess matrix elements. This is an important feature when polarization is needed for exploring a certain type of underlying dynamics. This option would be unavailable with the fixed order (FO) diagrammatic calculations [27-29]. In addition, the FO calculations may face a tremendous challenge for numerical stability dealing with the large collinear logs $\ln \left(Q^{2} / m_{\ell}^{2}\right)$.

(c) Although no logarithmic growth for the longitudinally polarized gauge boson PDFs, the large Yukawa coupling to the top quark and the scalar self-interaction of the Goldstone bosons make the subprocesses substantially enhanced, as seen for the VBF production of $t \bar{t}, t \bar{t} H, Z H$ and $H H$.

(d) For the PDFs of fermions with a bare SU(2) charge, due to the incomplete cancellation of the infrared divergence, they are not exactly factorizable. This is known as the violation of the Bloch-Nordsieck theorem $[15,20]$. This does not pose a problem to the beam (valence) lepton because it is a numerically small higher-order correction. This could lead to an unphysical solution to the dynamically generated neutrinos. We impose an infrared cutoff as a regulator $\tau^{\max }=1-M_{Z} / Q$, which assures the resummation to a double-log accuracy [16].

(e) We have not taken into account the effects of the finalstate radiations (FSR). This could become one of the dominant features at very high energies, properly described by the "fragmentation functions" $[15,30]$. We leave this topic for future explorations.

(f) We did not quantify the potentially large corrections near the threshold $Q^{2} \geq 4 m^{2}$. On the one hand, our formalism aims to address the physics far above the threshold $Q^{2} \gg M_{Z}^{2}$. On the other hand, the infrared behavior of the gauge boson radiation tends to populate the events in the low- $Q^{2}$ region. We leave this topic for future investigations.

(g) Although we focused on a $\mu^{+} \mu^{-}$collider in our presentation, the EW PDF formalism is equally applicable for $e^{+} e^{-}$colliders. The only difference is the QED radiation effects, further enhanced by a factor $\ln \left(m_{\mu}^{2} / m_{e}^{2}\right)$. It is also straightforward to apply our formalism to the high energy hadron colliders, although the photon PDF of the proton at a low scale is more subtle [31,32].

In summary, we advocated that all particles accessible under the SM interactions should be viewed as EW partons in high energy leptonic collisions well above the EW scale. We presented a systematic approach to define the EW PDFs for leptons and gauge bosons accurate to the order of LL under the unbroken gauge symmetry. We calculated the production cross sections for some characteristic SM processes at a high-energy muon collider in the EW PDF formalism. Polarized partonic cross sections can be evaluated individually that are desirable for exploring new physics beyond the Standard Model at future high energy colliders.

\section{ACKNOWLEDGMENTS}

We thank Christian Bauer, John Collins, Fabio Maltoni, Dave Soper and Bryan Webber for helpful discussions. This work was supported in part by the U.S. Department of Energy under Grant No. DE-FG02- 95ER40896, U.S. National Science Foundation under Grant No. PHY1820760, and in part by the PITT PACC. 
[1] E. S. for Particle Physics Preparatory Group, Physics briefing book, arXiv:1910.11775.

[2] P. Bambade, T. Barklow, T. Behnke, M. Berggren, J. Brau, P. Burrows, D. Denisov, A. Faus-Golfe, B. Foster, K. Fujii et al., The International Linear Collider: A global project, arXiv:1903.01629.

[3] P. Roloff, R. Franceschini, U. Schnoor, and A. Wulzer, The Compact Linear $e^{+} e^{-}$Collider (Clic): Physics Potential, arXiv:1812.07986.

[4] J. P. Delahaye, M. Diemoz, K. Long, B. Mansouli, N. Pastrone, L. Rivkin, D. Schulte, A. Skrinsky, and A. Wulzer, Muon colliders, arXiv:1901.06150.

[5] A. Collaboration, Towards an Advanced Linear International Collider, arXiv:1901.10370.

[6] C. F. von Weizsacker, Z. Phys. 88, 612 (1934).

[7] E. J. Williams, Phys. Rev. 45, 729 (1934).

[8] M. Greco, T. Han, and Z. Liu, Phys. Lett. B 763, 409 (2016).

[9] Y. L. Dokshitzer, Sov. Phys. JETP 46, 641 (1977).

[10] V. N. Gribov and L. N. Lipatov, Yad. Fiz. 15, 781 (1972) [Sov. J. Nucl. Phys. 15, 438 (1972)].

[11] G. Altarelli and G. Parisi, Nucl. Phys. B126, 298 (1977).

[12] H. Spiesberger, Phys. Rev. D 52, 4936 (1995).

[13] M. Roth and S. Weinzierl, Phys. Lett. B 590, 190 (2004).

[14] A. D. Martin, R. G. Roberts, W. J. Stirling, and R. S. Thorne, Eur. Phys. J. C 39, 155 (2005).

[15] J. Chen, T. Han, and B. Tweedie, J. High Energy Phys. 11 (2017) 093.

[16] C. W. Bauer, N. Ferland, and B. R. Webber, J. High Energy Phys. 08 (2017) 036.

[17] C. W. Bauer and B. R. Webber, J. High Energy Phys. 03 (2019) 013.
[18] T. Han, Y. Ma, and K. Xie (to be published).

[19] M. Ciafaloni, P. Ciafaloni, and D. Comelli, Phys. Rev. Lett. 84, 4810 (2000).

[20] M. Ciafaloni, P. Ciafaloni, and D. Comelli, Phys. Rev. Lett. 88, 102001 (2002).

[21] A. V. Manohar and W. J. Waalewijn, J. High Energy Phys. 08 (2018) 137.

[22] G. L. Kane, W. W. Repko, and W. B. Rolnick, Phys. Lett. 148B, 367 (1984).

[23] S. Dawson, Nucl. Phys. B249, 42 (1985).

[24] G. Cuomo, L. Vecchi, and A. Wulzer, SciPost Phys. 8, 078 (2020).

[25] P. Ciafaloni and D. Comelli, J. High Energy Phys. 11 (2005) 022.

[26] M. Ciafaloni, P. Ciafaloni, and D. Comelli, Phys. Rev. Lett. 87, 211802 (2001).

[27] A. Costantini, F. D. Lillo, F. Maltoni, L. Mantani, O. Mattelaer, R. Ruiz, and X. Zhao, J. High Energy Phys. 09 (2020) 080.

[28] J. Alwall, R. Frederix, S. Frixione, V. Hirschi, F. Maltoni, O. Mattelaer, H. S. Shao, T. Stelzer, P. Torrielli, and M. Zaro, J. High Energy Phys. 07 (2014) 079.

[29] W. Kilian, T. Ohl, and J. Reuter, Eur. Phys. J. C 71, 1742 (2011).

[30] C. W. Bauer, D. Provasoli, and B. R. Webber, J. High Energy Phys. 11 (2018) 030.

[31] A. Manohar, P. Nason, G. P. Salam, and G. Zanderighi, Phys. Rev. Lett. 117, 242002 (2016).

[32] B. Fornal, A. V. Manohar, and W. J. Waalewijn, J. High Energy Phys. 05 (2018) 106. 